\title{
Name relearning in elderly patients with schizophrenia: Episodic and temporary, not semantic and permanent
}

\author{
Tejinder K. Kondel \\ Cambridgeshire and Peterborough NHS Trust, UK. \\ Steven R. Hirsch \\ Imperial College, London, UK \\ Keith R. Laws \\ Nottingham Trent University, UK
}

\begin{abstract}
Introduction. Recent reports of lexical-semantic deficits in patients with schizophrenia (Laws, Al-Uzri, \& Mortimer, 2000; Laws, McKenna, \& Kondel, 1998). These studies suggest that younger patients have problems accessing intact memories and older patients show apparent "loss" of the lexical-semantic memory representations themselves.

Methods. Picture naming for everyday items was examined in a unique series of elderly patients with schizophrenia $(n=10)$ with a mean illness duration of 45.5 years; and compared with that in patients with probable Alzheimer's disease $(n=18)$ and elderly healthy controls $(n=27)$. Naming consistency across time was used as an indicator of whether the schizophrenic patients had difficulty accessing representations or a loss of the representations themselves. Finally, we examined the ability of the schizophrenic patients to relearn the names of unnamed items across four weekly retraining sessions and to retain them at a one month follow-up. Results. The elderly schizophrenic patients were as anomic as patients with probable Alzheimer's disease. Consistency analysis revealed that the patients had storage deficits. Analysis of patient error types was consistent with a semantic deficit. Finally, the schizophrenic patients showed significant improvement with relearning, but this was not maintained at follow-up.
\end{abstract}

Correspondence should be addressed to Dr Keith R. Laws, Brain and Cognition Research Group, Division of Psychology, Nottingham Trent University, Burton Street, Nottingham, NG1 4BU, UK; email: keith.laws@ntu.ac.uk

Part of this study was presented at the Biennial Winter Workshop in Schizophrenia and TKK would like to thank the conference organisers for awarding her a Young Scientist Award; it was also presented at the 10th Association of European Psychiatrists Congress. This research was supported by the Stanley Foundation. Thanks finally to the patients and the staff at "the Limes" for their help with the testing.

(C) 0000 Psychology Press Ltd

http://www.tandf.co.uk/journals/pp/13546805.html

DOI:10.1080/13546800444000137 
Conclusions. Elderly patients with schizophrenia show a profound and stable anomia. Although name relearning induced some significant gains in naming, these were short-term and reflect episodic rather than semantic reinstatement of representations. Implications for cognitive remediation are discussed.

Recent studies point to a lexical-semantic disorder in patients with schizophrenia (Al-Uzri, Laws, \& Mortimer, in press; Laws et al., 1998, 2000). For example, Laws et al. (2000) reported that picture naming was significantly impaired in schizophrenic subjects when compared with data from 500 normal subjects. Moreover, this impairment was disproportionate to any general intellectual deficit; and comparable in both degree and type to the naming problems found in a cohort of neurological patients with left hemisphere lesions.

Criteria derived from the neurological literature (see Warrington \& Shallice 1979 and Figure 1) have been used to examine whether such lexical-semantic deficits in patients with schizophrenia reflect a loss/degradation of underlying representations or a problem with accessing intact representations (Al-Uzri et al., in press; Laws et al, 1988, 2000; Leeson, McKenna, \& Laws, in press). In other words, are the representations intact, but inaccessible or are they "lost" or degraded. A recent review of relevant studies (Al-Uzri, in press) suggests that more access-like or even normal picture naming occurs in younger patients with schizophrenia (Al-Uzri et al., in press), while storage-like naming deficits are more typical of older patients (Laws et al., 1998, 2000). A recent cross-sectional study of 56 patients with a wide range of illness length (3-40 years) indicates a slightly more complex position, with an initial quite large lexical degradation

\begin{tabular}{|c|c|}
\hline Access Disorder & Store Disorder \\
\hline $\begin{array}{l}\text { Superordinate information equivalent to attribute } \\
\text { information } \\
\text { Can state that person is a 'politician' and that he is 'prime } \\
\text { minister' }\end{array}$ & $\begin{array}{l}\text { Superordinate information better preserved than } \\
\text { attribute information } \\
\text { Can state that person is a 'politician' but not that he is } \\
\text { 'prime minister' }\end{array}$ \\
\hline $\begin{array}{c}\text { Inconsistent responses } \\
\text { Can sometimes retrieve the name 'Tony Blair' }\end{array}$ & $\begin{array}{l}\text { Consistent responses } \\
\text { Never or always retrieves the name 'Tony Blair' }\end{array}$ \\
\hline $\begin{array}{l}\text { No frequency of familiarity effect } \\
\text { Finds it equally difficult to name 'Tony Blair' and } \\
\text { 'Richard Briers' }\end{array}$ & $\begin{array}{c}\text { Frequency or familiarity effect } \\
\text { Better able to name 'Tony Blair' than 'Richard Briers' }\end{array}$ \\
\hline $\begin{array}{c}\text { Cueing effect } \\
\text { Helped by cues such as 'Is the current prime minister' or } \\
\text { 'Name is Tony }\end{array}$ & $\begin{array}{c}\text { No cueing effect } \\
\text { Not helped by cues such as 'Is the current prime minister' or } \\
\text { 'Name is Tony }\end{array}$ \\
\hline
\end{tabular}

Figure 1. Criteria for determining disorders of access and storage. 
occurring quite early in the disease, which is then followed by a gradual continual depletion of storage and access procedures (Leeson et al., in press).

One of the criteria for examining this issue concerns the fact that patients with access problems should benefit from cueing, while those with store problems should not benefit from any cues because representations have been "lost". In this context, we examined picture naming in a unique cohort of patients with schizophrenia all of whom had very long lengths of illness, with the minimum being 42 years. To our knowledge, no previous study of lexicalsemantic functioning has investigated such elderly patients (who would be prime candidates for showing severe lexical storage deficits). The current study uses an individual case study approach to determine the effect of reteaching the names of previously unnamed items to the patients (i.e., using the whole name as a cue). Each patient received retraining on four occasions (once per week for one month) and then were retested on a follow-up occasion (one month after the final testing) to see what was retained. If these elderly chronic patients have undergone a depletion of their lexical-semantic store, then we might expect retraining to have little long-term benefit, though short-term benefits may accrue through episodic relearning; however, if they have access problems (with intact representations) then retraining may be helpful in the short- and long-term.

\section{METHODS}

\section{Participants}

Chronic schizophrenics. Ten chronically hospitalised elderly patients with schizophrenia (mean age 75 years; mean length of illness 45.05 years; range 42-52 years) were drawn from a sample of chronically hospitalised psychiatric patients. The initial diagnoses were made over 40 years ago, so it is difficult to relate to current opinion in any meaningful manner. Nevertheless, patients were included in the study only if two psychiatrists currently and independently confirmed a diagnosis of schizophrenia at the time of testing. Diagnosis was based upon clinical interview; however, no symptom ratings were recorded. Additionally, patients were included only of there was no history of head injury, neurological problems, comorbid diagnosis (including dementia and depression), and alcohol or drug abuse. The mean Mini Mental State Examination (MMSE) score of the patients was 19.6 and their mean NART was 102.2.

DAT patients. Eighteen patients (age $77.5 \pm 7.5$ ) diagnosed with probable Alzheimer's disease according to NINCDS-ADRDA criteria (McKhann et al., 1984) were tested at baseline. All were living at home and visiting a day centre. Their mean MMSE of 18.03 suggests moderate impairment.

Healthy elderly normal controls. Twenty-seven normal control participants aged $65+$ (mean age $72.35 \pm 4.9$ ) were tested at baseline. They were recruited 
through their general practitioner, who screened them for good health. They had no history of head injury, neurological or psychiatric illness, and alcohol or drug abuse. The mean estimated NART IQ was 109.

\section{Procedure}

Line drawings of 64 highly familiar everyday items (e.g., apple, dog, bicycle, button, see Figure 2) were selected from the Snodgrass and Vanderwart corpus (1980). Each item was sized to a minimum of $10 \mathrm{~cm} \times 10 \mathrm{~cm}$ and printed on a sheet of A4 paper. The images were presented one at a time for confrontation naming without time limit.

\section{Results}

Degree of anomia. Naming of simple line drawings was extremely impaired in the group of chronic elderly patients (mean $44 \pm 12.1,69 \%$ ) when compared to controls $(61.47 \pm 1.95,96 \%)$, but comparable with that of Alzheimer patients $(40.5 \pm 17.5,63 \%)$. The patients with schizophrenia had significantly worse
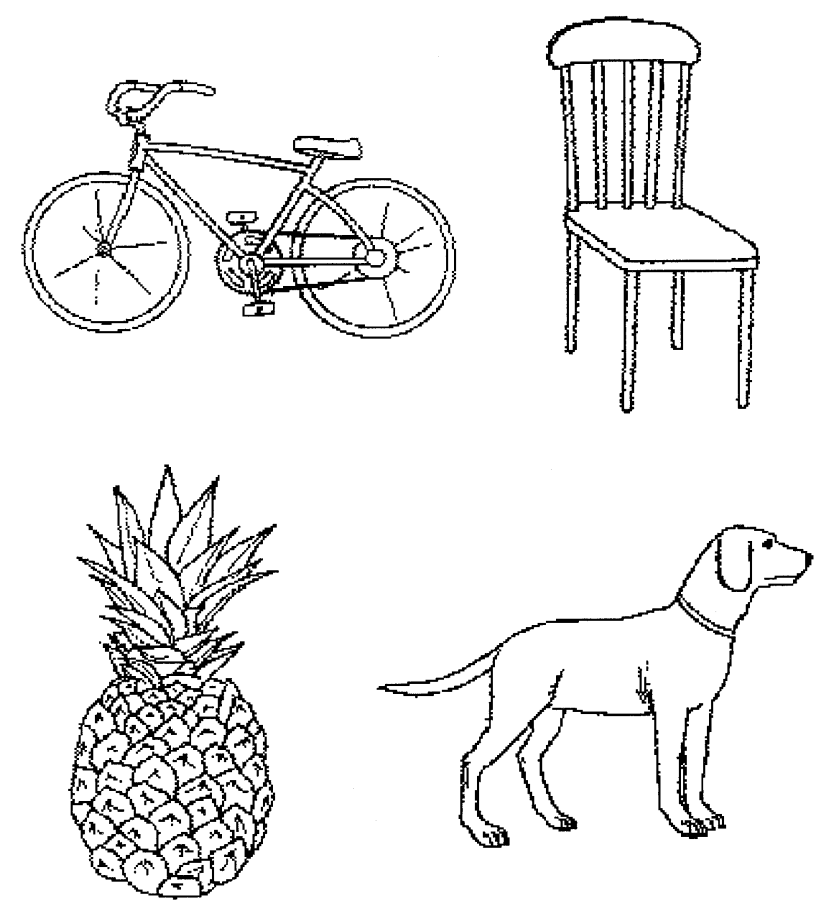

Figure 2. Examples of stimuli presented for naming (from the Snodgrass \& Vanderwart 1980 linedrawing corpus). 
naming than normal controls $(t(35)=6.64, p<.0001$; the effect size was very large, $d=2.75)$ as did the Alzheimer patients $(t(43)=7.95, p<.0001)$. Indeed, all of the patients with schizophrenia fell more than 2.5 standard deviations below the mean naming score for the healthy controls. Moreover, the mean for these patients was not significantly different to that for the DAT patients $(t(26)=$ $0.27, p=.79$; the effect size was small, $d=0.22$ ).

To determine their level of receptive vocabulary, the patients with schizophrenia were tested on the short-form of the British Picture Vocabulary Scale (Dunn, Dunn, Whetton, \& Pintilie, 1982); A word-picture matching task. Their raw mean score of $16.55(S D=7.5)$ was severely impaired according to test norms $(<1$ st percentile for 16-year-olds). This score also correlated highly and significantly with their baseline naming scores $(\mathrm{r}=.62, p<.05)$.

Access and storage probabilities. Naming in 9 of the 10 patients with schizophrenia was retested one month after the baseline naming session. These data were analysed using the two-parameter stochastic model of Faglioni and Botti (1993) to determine whether the schizophrenic patients showed storage or access difficulties. This analysis examines naming consistency across time to provide two probabilities: A probability that a name is stored $(s)$ and the other being the probability that a stored name will be retrieved $(r)$. In essence, the value of $(s)$ sets an upper limit on the number of items that are available and $(r)$ determines the number of stored items that are accessible. The values of $(s)$ and $(r)$ are calculated using:

$$
\begin{aligned}
& \text { Retrieval probability }(r)=2 s_{2} /\left(s_{1}+2 s_{2}\right) \\
& \text { Storage probability }(s)=\left(s_{1}+2 s 2\right) / 2 r
\end{aligned}
$$

As might be expected, it revealed a wide range of storage and retrieval probabilities; however, it is clear that most patients have poor storage but retrieve most of the stored items.

Analysis of consistency across time revealed that the estimated storage size for all schizophrenic patients was lower than the retrieval for any healthy control; and in four cases, worse than the mean retrieval in Alzheimer's patients (see Figure 3). There was no significant difference between the estimated numbers of names stored and those retrieved by the schizophrenic patients $(\mathrm{F}<1)$. The major difference amongst the schizophrenic patients occurred on the storage dimension (range .16-.86); the retrieval dimension revealed little difference (only GC had a retrieval probability that was less than .8; the remaining range was .82-.97)

Analysis of error types. The naming errors made by the patients with schizophrenia were analysed further according to type (see Laws, 2000). All errors were classified into one of the following categories by three subjects; in 


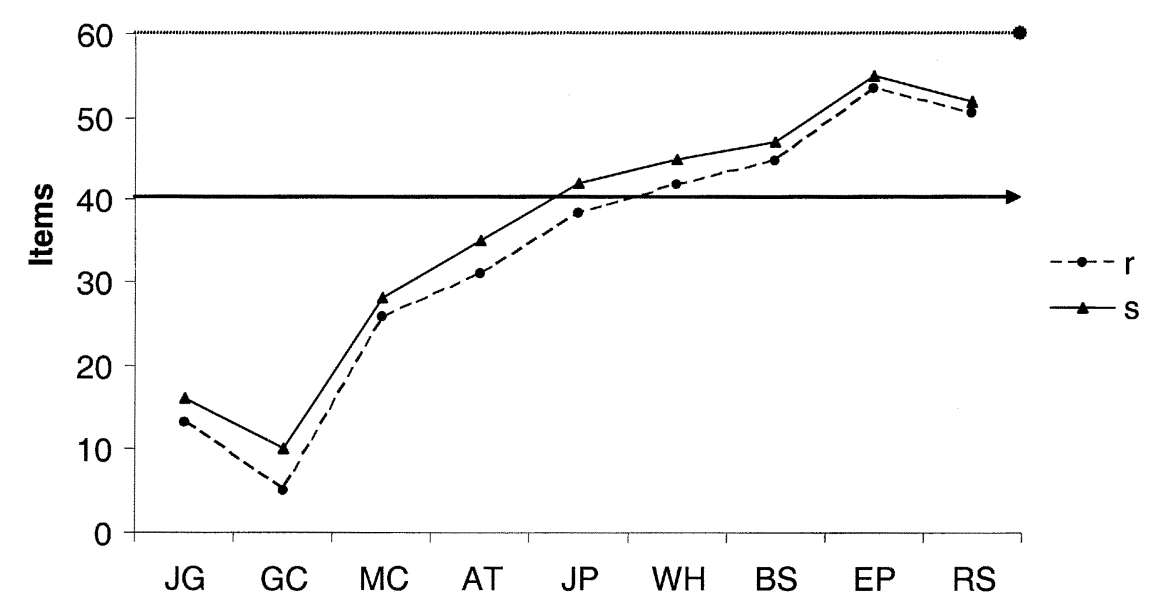

Figure 3. Estimated number of item names $(n=64)$ that are stored and retrievable in elderly patients with schizophrenia. The broken line represents the mean retrieval of healthy controls, while the unbroken line represents the retrieval of Alzheimer's patients.

the vast majority of cases, there was a unanimous decision; the remainder were on the basis of majority decisions.

1. Visual errors: responses similar to the target and from a different semantic category (e.g., Tent for Mountain).

2. Ambiguous visual/semantic category errors: responses from the same category as the target and visually similar such that the error could be semantically or visually based (e.g., Hippopotamus for Rhino; Peanut for Acorn).

3. Semantic within-category errors: responses from the same semantic category but not visually similar (e.g., Hammer for Chisel; Violin for Accordion).

4. Semantic superordinate errors: responses giving general class (e.g., Animal for Zebra; musical instrument for Violin).

5. Phonemic errors: mispronunciations or distortions of target name sharing at least one syllable (e.g., Iglow for Igloo; Proporactor for Protractor).

6. Nonresponse: includes "don't know" and nonresponses.

7. Others: unrelated errors or bizarre responses.

It is notable that the proportions of "nonresponses" and "don't know" answers were low, indicating that the patients more often made an attempt to name the item than to simply give no response (see Table 1). Similarly, the number of phonemic errors was zero and the number of "other" (e.g., bizarre) responses was low (except in the case of WH). Although we cannot be sure of the focus of 
TABLE 1

Proportions of different error types made by patients with schizophrenia

\begin{tabular}{lrrrrrrr}
\hline Patient & Superordinate & Semantic & Visual & $\begin{array}{c}\text { Visual- } \\
\text { semantic }\end{array}$ & $\begin{array}{r}\text { Nonresponse } \\
\text { Don't know }\end{array}$ & Phonemic & Others \\
\hline JG & 0.00 & 32.56 & 25.58 & 18.60 & 20.93 & 0.00 & 2.33 \\
GC & 0.00 & 22.00 & 12.00 & 22.00 & 34.00 & 0.00 & 10.00 \\
MC & 0.00 & 17.24 & 44.83 & 31.03 & 0.00 & 0.00 & 6.90 \\
AT & 18.18 & 18.18 & 27.27 & 13.64 & 18.18 & 0.00 & 4.55 \\
JP & 0.00 & 5.56 & 22.22 & 38.89 & 27.78 & 0.00 & 5.56 \\
WH & 0.00 & 23.08 & 7.69 & 30.77 & 0.00 & 0.00 & 38.46 \\
BS & 8.33 & 16.67 & 16.67 & 41.67 & 16.67 & 0.00 & 0.00 \\
EP & 0.00 & 25.00 & 50.00 & 25.00 & 0.00 & 0.00 & 0.00 \\
RS & 22.22 & 22.22 & 22.22 & 11.11 & 22.22 & 0.00 & 0.00 \\
Mean & 5.42 & 20.28 & 25.39 & 25.86 & 15.53 & 0.00 & 7.53 \\
\hline
\end{tabular}

the deficit by examining error types, the finding that patients mostly made semantic, visual and visual-semantic errors is consistent with a semantic level difficulty. They tended, for example, to choose the names of related items (see examples in Table 2) rather than to give superordinate names.

\section{NAME RETRAINING}

One month after the initial testing, we examined the effect of reteaching the names of any unnamed items to the patients with schizophrenia.

TABLE 2

Three examples of errors made by each patient (Target $\rightarrow$ Response)

\begin{tabular}{lrrr}
\hline Patient & & & \\
\hline JG & Banana $\rightarrow$ Peach & Pineapple $\rightarrow$ Strawberry & Ostrich $\rightarrow$ Lion \\
GC & Kangaroo $\rightarrow$ Wolf & Cherry $\rightarrow$ Carrot & Pineapple $\rightarrow$ Carrot \\
MC & Sled $\rightarrow$ Tadpole & Frog $\rightarrow$ Tortoise & Helicopter $\rightarrow$ Plane \\
AT & Helicopter $\rightarrow$ Plane & Frog $\rightarrow$ Animal & Pineapple $\rightarrow$ Fruit \\
JP & Celery $\rightarrow$ Spinach & Ostrich $\rightarrow$ Peacock & Pineapple $\rightarrow$ d/k \\
WH & Zebra $\rightarrow$ Giraffe & Pineapple $\rightarrow$ Grapefruit & Chisel $\rightarrow$ Brush \\
BS & Helicopter $\rightarrow$ Aeroplane & Plug $\rightarrow$ d/k & Tomato $\rightarrow$ Apple \\
EP & Celery $\rightarrow$ Artichoke & Nail $\rightarrow$ Screw & Fox $\rightarrow$ Wolf \\
RS & Goat $\rightarrow$ d/k & Cherry $\rightarrow$ Fruit & Plug $\rightarrow$ Electrics \\
\hline
\end{tabular}

$\mathrm{d} / \mathrm{k}=$ ? 


\section{Method and procedure}

Each of the nine patients received four weekly individual retraining sessions and this consisted of presenting the 64 items for naming. For those items that they could not name, the experimenter spoke aloud the name and asked the patient to repeat it back twice. Finally, they were given a surprise follow-up retest one month after the final training session.

\section{Results}

An ANOVA with the four levels of time as the repeated measures factor revealed a significant linear improvement in naming (see Figure 4) between week 1 and week $4, F(3,24=11.24, p<.0001)$. All patients, except one (BS), showed some improvement (see Table 3). After 4 weeks of retraining, the patients were still impaired when compared to the healthy controls, although the effect size had almost halved $(d=-1.47)$.

To ensure that any changes reflected retraining rather than regression to the mean or natural performance variability, we determined the amount of natural fluctuation for each subject between the initial baseline and that before training started four weeks later. A repeated measures t-test revealed a significant improvement in naming over that period of one month $t(8)=2.4, p=.043: 39.11$ vs. 42.44). The change across one month was taken as an indicator of normal

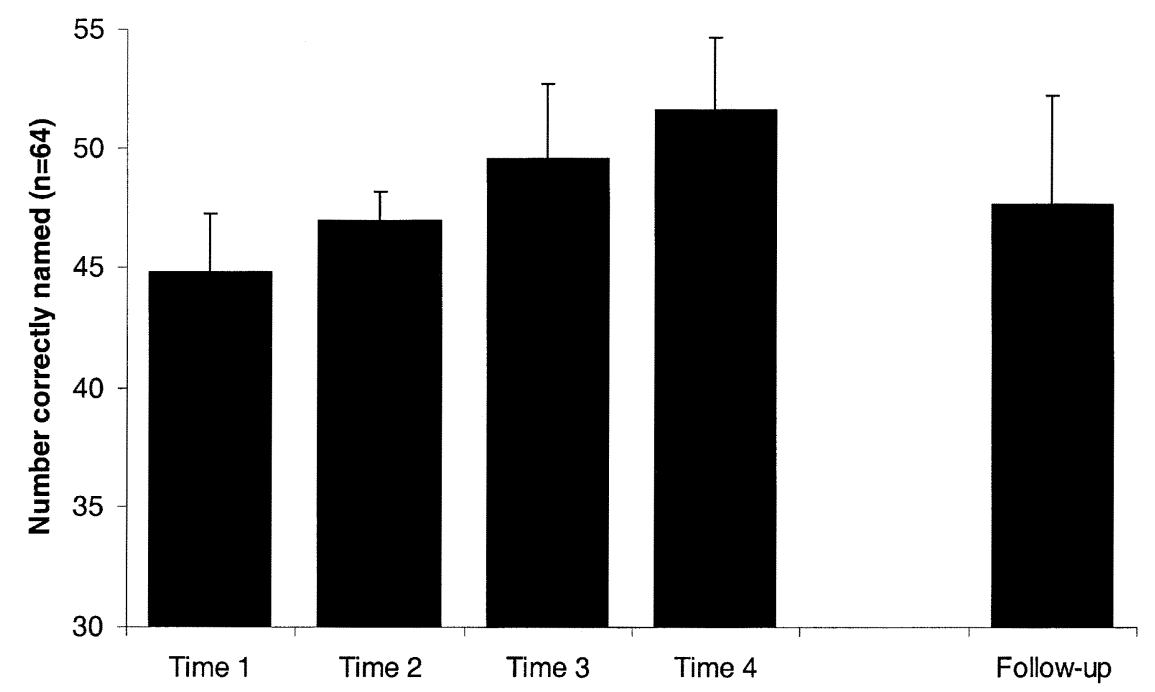

Figure 4. Naming performance for patients with schizophrenia during retraining and follow-up. There is a linear improvement in naming across retraining sessions, but that this was not sustained at one month follow-up. Bars indicate standard deviation adjusted to remove the between-subject variance in a repeated measures design (see Loftus \& Masson, 1994). 
TABLE 3

Number of items named correctly after each weekly name retraining

\begin{tabular}{lllllll}
\hline Patient & Time 1 & Time 2 & Time 3 & Time 4 & Follow-up & $\begin{array}{c}\text { Change from } \\
\text { baseline }\end{array}$ \\
\hline JG & 23 & 29 & 33 & 30 & 28 & 5 \\
GC & 28 & 30 & 33 & 35 & 36 & 8 \\
MC & 36 & 37 & 36 & 41 & 32 & -4 \\
AT & 45 & 50 & 59 & 61 & 40 & -5 \\
JP & 49 & 48 & 49 & 57 & 51 & 3 \\
WH & 54 & 55 & 55 & 60 & 60 & 6 \\
BS & 55 & 55 & 57 & 55 & 55 & 9 \\
EP & 55 & 61 & 63 & 64 & 64 & 3 \\
RS & 58 & 57 & 61 & 61 & 61 & 0 \\
Mean & 44.78 & 46.89 & 49.56 & 51.56 & 47.56 & \\
\hline
\end{tabular}

fluctuation in their naming. The amount of improvement with training was significantly larger than that occurring through natural fluctuation and thus, does not reflect a regression to the mean $t(8)=2.38, p=.044: 6.78 \pm 4.41 \mathrm{vs} .2 .33 \pm$ 2.92). Indeed, the almost perfect correlation between initial baseline and week 1 $(r=.98)$ indicates that regression to the mean is highly unlikely $(=2 \%$ regression to the mean)

Nevertheless, the critical question concerns whether any benefit is sustained at follow-up. A comparison between week 1 and follow-up was non-significant $t(8)=-1.66, p=.14)$, suggesting that the benefit was not sustained over the baseline. No relationship emerged between the baseline naming of the patients and the amount of change retained at follow-up $(r=-.06)$.

Reliable Change Index (RCI): Clinical significance. We used the Reliable Change Index (RCI; Jacobson \& Traux, 1991) to assess whether individual patients had shown a clinically significant improvement during name retraining. Jacobson and Traux (1991) recommended that each individual might be categorised as improved if the amount of change for that individual on a given measure exceeded chance expectations. In contrast to typical inferential statistics applied to evaluating the effects of intervention, the RCI is a psychometrically sound method of categorising individuals according to whether each has improved during the course of training.

$$
\mathrm{RCI}=(\text { mean } \mathrm{A}-\text { mean } \mathrm{B}) / S D \cdot \sqrt{1-r_{x x}}
$$

The RCI is the mean difference divided by the standard error of measurement (SE). A change for an individual that exceeds $1.96 \mathrm{SE}$ is unlikely to be merely a function of measurement error; conversely, if the RCI score is 1.96 or less, then 
any change may reflect the unreliability of the measure. The RCI revealed that a reliable change would be an improvement in naming of at least six items. At the end of the training five patients had shown a reliable improvement and at the follow-up, three (GC, WH, and $\mathrm{EP}$ ) continued to show a reliable change.

Compared to the baseline normal control data, six patients achieved at least one naming performance within the normal range $(57+)$ normal (these were the best six patients at baseline: (AT, JP, WH, BS, EP, and RS).

\section{DISCUSSION}

This study documents a severe anomia for simple everyday objects in elderly patients with schizophrenia. Moreover, the level of impairment was comparable with that seen in a sample of patients with probable Alzheimer's dementia. The finding of impaired comprehension on the British Picture Vocabulary Scale and its correlation with their naming performance, suggests that the patients with schizophrenia have a central semantic disorder rather than a simple expressive lexical deficit. The analysis of their naming errors was also consistent with this interpretation. The patients had low storage rather than low access probabilities indicating that retraining may not be easy or successful. Indeed, although the patients with schizophrenia showed linear improvements in their naming after weekly retraining sessions; at a one month follow-up their naming had returned to a level that was indistinguishable from the first week of training.

Turning first to possible confounding explanations for the poor naming in these elderly patients with schizophrenia. While this sample of patients have been hospitalised for an extremely long time (range 32-52 years), the choice of common and highly familiar stimuli, along with the near ceiling performance of matched controls, makes it untenable that the patients had never learned the item names. This latter point also seems unlikely given that patient NART scores point to average estimated premorbid intellectual functioning. It also seems unlikely that their poor naming reflects lowered motivation or lack of cooperation since the patients participated in testing across several weeks. While the elderly schizophrenic patients performed extremely poorly on the naming task at baseline, the improvement in their performance with retraining also cannot be explained as a product of regression to the mean. It might be argued that since the patients showed evidence of a comprehension deficit that correlates with their anomia, the former may inflate or even determine the latter. The most likely explanation is that both reflect a central semantic deficit affecting input (comprehension) and output (naming). While the comprehension problems may affect naming, the ability of the patients to relearn the names clearly indicates that they understood the task requirements. Finally, their poor naming does not appear to reflect any "known" comorbid dementia or depression. Certainly, none of the patients showed evidence of a "regular" dementia (i.e., unrelated to their schizophrenia) through clinical interview or in the opinion of their long- 
term carers. Although we cannot eliminate a degenerative atrophy, their moderately poor MMSE scores (mean 19.6) plus the evidence of good short-term memory learning do not point strongly to the presence of a regular dementia.

These data raise some interesting questions concerning whether these elderly patients have degraded (lost) representations or poor access to those representations; and what types of representations are being affected. On the one hand, the patients show some natural and significant change in their naming across time (one month). Additionally, however, they showed significant change (improvement) following retraining. While performance variability may be associated with item accessibility, these two types of variability are very different and may reflect quite different underlying sources. The first-natural fluctuation-may reflect changes in the accessibility of the semantic-lexical network (i.e., the extent to which extant concepts and names are activated in lexical semantics on presentation of the appropriate picture). Indeed, the relearned names relate to highly familiar items (and therefore resistant to degradation and loss) and so, viewed as evidence of partial preservation of the concepts underlying the items (see Snowden \& Neary, 2002). The names (re)acquired through relearning do not necessarily, however, reflect output from the semantic system, but may indicate temporary changes in the episodic memory network. For example, the patients might be simply forming an episodic paired association, that is, between the image and the word (and this may even be arbitrary for them if they have no semantic substrate for this). Given that the items were again unavailable at a surprise one month follow-up (thus, the patients had not been rehearsing), it suggests that even if the underlying semantic network is partly intact, it is clearly insufficient to support simple concepts in the longer-term. By contrast, we know that their episodic memory can support temporary improvements in naming, so it would appear that the improved naming in at least some of these patients is an episodic phenomenon (largely dependent on practice and rehearsal).

At the level of individual patients, some (3/9: GC, WH, and EP) did show a clinically significant retraining benefit at follow-up. Two (WH and EP) even normalised after training, although both were initially performing close to the level of healthy controls. By contrast, the other (GC) was much more impaired (and had the smallest retrieval probability of the cohort at .51 and so, may be presenting with access problems). Hence, the amount of change and the ability to sustain improvement was unrelated to level of baseline performance. The range of naming performance reported here is typical of the heterogeneous cognitive performance in schizophrenics generally (see Laws et al., 1998), and was quite similar to the level of spread seen in the Alzheimer patients. It does, however, indicate a variable response to retraining in severely impaired patients (most will show change, but only some will continue to show this in the longterm). It is also worth noting patient AT, who showed the largest gain with retraining and then returned to a level that was lower than the baseline. The 
degree to which patients are able to sustain retraining benefits in the longer-term may well reflect the integrity of the underlying semantic substrate (and absolute naming ability is therefore not necessarily a good indicator of this).

This pattern accords with the conclusions of a recent review (Al-Uzri, in press) showing that consistent storage-like naming deficits in older schizophrenic patients and more access-like or even normal picture naming in younger patients. The findings go further than simply saying that factors, such as age and chronicity, are associated with storage problems. The data presented here show that most of these elderly schizophrenic patients display a severe anomia, that has storage characteristics; however, the patients also exhibit some short-term episodic benefit from retraining and so, are able to acquire new (or reacquire lost) information (but only in the short-term). Nevertheless, this is an episodic rather than semantic phenomenon. In terms of implications for cognitive rehabilitation, the current study indicates that short-term relearning benefits are possible in older patients, but these may be transient because they constitute episodic relearning rather than semantic integration (or reintegration). Obviously, one implication is that longer-term semantically based benefits are more likely to result from interventions that occur earlier in the course of this illness.

Manuscript received 19 January 2004 Revised manuscript received 10 June 2004

\section{REFERENCES}

Al-Uzri, M.M. (in press). Can neuroplasticity improve our understanding of semantic memory deficit in schizophrenia? History and Philosophy of Psychology.

Al-Uzri, M.M., Laws, K.R., \& Mortimer, A.M. (in press). An early marker for semantic memory impairment in patients with schizophrenia. Cognitive Neuropsychiatry

Dunn, L.M., Dunn, L.M., Whetton, C., \& Pintilie, D. (1982). British picture vocabulary scale. Nelson NFER.

Faglioni, P., \& Botti, C. (1993). How to differentiate retrieval from storage deficit: A stochastic approach to semantic memory modelling. Cortex, 30, 501-518.

Jacobson, N., \& Truax, P. (1991). Clinical significance: a statistical approach to defining meaningful change in psychotherapy research. Journal of Consulting and Clinical Psychology, 59, 12-19.

Laws, K.R. (2000). Category-specific naming errors in normal subjects: the role of evolution and experience. Brain and Language, 75, 123-133.

Laws, K.R., Al-Uzri, M.M., \& Mortimer, A.M. (2000). Lexical knowledge degradation in schizophrenia. Schizophrenia Research, 45, 123-131.

Laws, K.R., McKenna, P.J., \& Kondel, T.K. (1998). On the distinction between access and store deficits in schizophrenia: a question of deficit severity. Neuropsychologia, 36, 313-321.

Leeson, V.C., McKenna, P.J., \& Laws, K.R. (in press). Storage and access procedures in schizophrenia: evidence for a two-phase model of lexical impairment. Journal of Clinical and Experimental Neuropsychology

Loftus, G.R., \& Masson, M.E.J (1994). Using confidence intervals in within-subject designs. Psychonomic Bulletin and Review, 1, 476-490. 
McKhann, G., Drachman, D., Folstein, M., Katzman, R., Price, D., \& Stadklan, E.M. (1984). Clinical diagnosis of Alzheimer's disease: report of the NINCDS-ADRDA work group under the auspices of Department of Health and Human Services Task Force on Alzheimer's Disease. Neurology, 34, 939-944.

Snodgrass, J.G., \& Vanderwart, M (1980). A standardised set of 260 pictures: norms for name agreement, image agreement, familiarity and visual complexity. Journal of Experimental Psychology: Human Learning and Memory, 6, 174-215.

Snowden, J.S., \& Neary, D. (2002). Relearning of verbal labels in semantic dementia. Neuropsychologia, 40, 1715-1728.

Warrington E. K., \& Shallice T. (1979). Semantic access dyslexia. Brain, 102, 43-63. 


\section{Cognitive Neuropsychiatry}

\section{AUTHOR QUERIES}

(2217) Kondel et al.

Please note that page numbers refer to printed pages

Q1 2 Al-Uzri et al. (in press) $\times$ 3: any update?

Q2 2 Leeson et al. (in press) $\times 2$ : any update?

Q3 3 NART: please provide in full

Q4 3 DAT: please provide in full

Q5 10 the text is not clear?

Q6 12 Al-Uzri (in press): any update?

\section{References}

Al-Uzri (in press): any update? Al-Uzri et al. (in press): any update? Leeson et al. (in press): any update?

Snodgrass \& Vanderwart (1980): please check the 'subtitle' of the journal

\section{Table 2}

$\mathrm{d} / \mathrm{k}$ : please provide this in full in a footnote 